\title{
Benchmarking of a 3D non-linear lifting line method against 3D RANSE simulations
}

\author{
Chloé Duport $^{1, *}$, Jean-Baptiste Leroux ${ }^{1}$, Kostia Roncin ${ }^{1}$, Christian Jochum ${ }^{1}$ and Yves Parlier ${ }^{2}$ \\ ${ }^{1}$ ENSTA Bretagne, IRDL, UMR CNRS 6027, 2 rue François Verny, 29806 Brest Cedex 9, France \\ ${ }^{2}$ Beyond the sea, 1010 avenue de l'Europe, 33260 La Teste de Buch, France
}

\begin{abstract}
As a part of the design and operation of kites as auxiliary propulsion of vessels, it is necessary to be able to quickly estimate the aerodynamic efforts along various trajectories. A 3D non-linear model based on the lifting line of Prandtl has been developed for this purpose. It allows these rapid calculations for wings with any laws for the dihedral angle, the twist, and the sweep angle, along the span, and for a general flight kinematic taking into account translation velocities and rotation rates. This model has been verified by comparison with 3D simulations performed with a Navier-Stokes solver. It gives satisfactory results in incidence and sideslip, with gaps of about $4 \%$ for forecasts lift. Special attention has been paid to the estimation of the accuracy of the provided numerical results.
\end{abstract}

Keywords: 3D non-linear lifting line / kite / aerodynamic forces / validation / RANSE simulations

Résumé - Comparaison d'une méthode de ligne portante 3D non linéaire avec des simulations RANSE 3D. Dans le cadre de la conception et de l'exploitation de cerfs-volants pour la propulsion auxiliaire de navires, il est nécessaire de pouvoir simuler rapidement les efforts aérodynamiques le long de trajectoires quelconques. Un modèle 3D non linéaire basé sur une extension de la ligne portante de Prandtl a été mis au point à cet effet. Il permet des calculs rapides pour des ailes avec des angles de dièdre, de vrillage et de flèche, variables en envergure, pour des cinématiques de vol prenant en compte vitesses de translation et taux de rotation. Ce modèle a été vérifié grâce à des simulations 3D réalisées avec un solveur Navier-Stokes. Il donne des résultats satisfaisants en incidence et en dérapage, avec par exemple des écarts d'environ $4 \%$ pour les prévisions en portance. Une attention particulière a été apportée à l'estimation de la précision des résultats numériques produits.

Mots clés : ligne portante 3D non linéaire / kite / chargement aérodynamique / vérification / simulations RANSE

\section{Introduction}

This work is part of a project which aims to develop tethered kite systems for auxiliary propulsion of merchant ships. Knowing the costs of experimental campaigns and the computational time needed to carry out the CFD simulation of a kite, it is necessary to develop simple and fast aerodynamic models, reliable enough to estimate the kite properties for general flight kinematics.

The Prandtl lifting line is a right method used for wings performances prediction. As an example, Graf et al. (2014) use a non-linear iterative lifting line method to predict the lift and drag of a two-element straight wing for an AC72 catamaran. The comparison with Reynolds Averaged Navier-Stokes

*Correspondence: chloe.duport@ensta-bretagne.org
Equations (RANSE) simulations shows a good agreement for attached flow regime. Leloup et al. (2013) adapt the lifting line method for 3D kite wings with variable dihedral and sweep angles along the span. However in this work a direct solving, based on a collocation method, is used, which makes it impossible to take into account the non-linearity of the lift coefficient. Another example is the non-linear method by Phillips and Snyder (2000), but adapted to straight wings in translation with constant sweep or constant dihedral angles.

This note presents in section 2 a new 3D non-linear lifting line method able to cope with wings when dihedral and sweep angles are great. In section 3, the RANSE simulations, carried out to validate the $3 \mathrm{D}$ non-linear lifting line results, are described, and then the comparative results are discussed. Some more details and an application case can be found in Duport et al. (2016). 


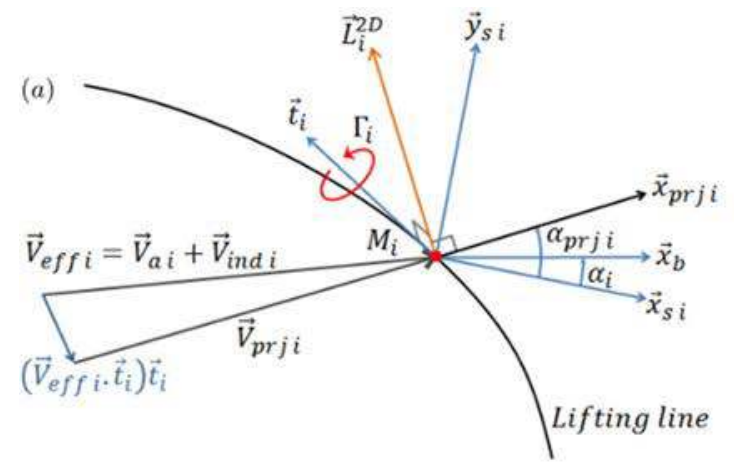

(b)

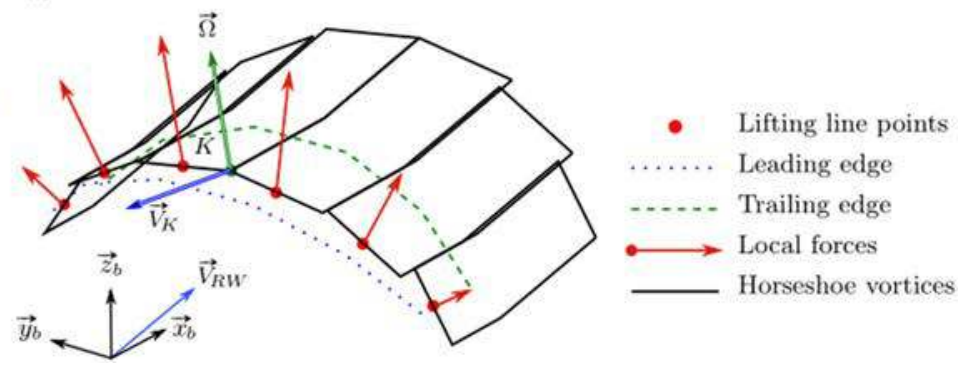

Fig. 1. a: local effective wind projected in the section plane $i$; b: example of a low discretized lifting line model.

\section{3D non-linear lifting line method}

The 3D non-linear lifting line is based on an extension of the Prandtl's lifting line theory, intended to address cases of wings with variable dihedral and sweep angles along the span, and to take into account the non-linearity of the lift coefficient. The kite wing is supposed to fly in a given wind $\vec{V}_{R W}$, with a solid kinematic torsor $\left\{\vec{V}_{K} \mid \vec{\Omega}\right\}$, where $K$ is the quarter chord point in the symmetry plane. The kite global apparent wind is $\vec{V}_{a}=\vec{V}_{R W}-\vec{V}_{K}$.

The kite geometry is defined in the Cartesian reference frame $\left(K, \vec{x}_{b}, \vec{y}_{b}, \vec{z}_{b}\right)$ (see Fig. 1). A generatrix line is given as a parametric planar curve $M(s)$ in the plane $\left(K, \vec{y}_{b}, \vec{z}_{b}\right)$, $M(s)$ being the quarter chord point of the current section. The section of the kite is defined in the normal plane $\left(M(s), \vec{x}_{b}, \vec{n}(s)\right)$ to this line, where $\vec{n}(s)=\vec{x}_{b} \Lambda \vec{t}(s)$ and $\vec{t}(s)$ is the vector tangent to the generatrix. The local chord direction $\vec{x}_{s}(s)$ is obtained from the rotation of a given twist law angle $\alpha(s)$ around $\vec{t}(s)$ of the vector $\vec{x}_{b}$. This finally leads to the local chord reference frame of the kite section $\left(M(s), \vec{x}_{s}(s), \vec{y}_{s}(s)\right)$ with $\vec{y}_{s}(s)=\vec{x}_{s}(s) \wedge \vec{t}(s)$, where the section points are placed, from a given section definition, scaled with a chord law $c(s)$. In the case of a kite wing with sweep angle, the local section points are finally translated by the vector $f(s) \vec{x}_{b}$, with $f(s)$ a given sweep law.

The finite wing and its wake are represented by a set of $n$ horseshoe vortices of different strengths $\Gamma=\left(\Gamma_{i}\right)_{i=1 \ldots n}$, derived from Katz and Plotkin (2001). Each bound vortex is located at the local quarter chord length, perpendicularly to the plane of the considered section. An example of a discretised model is presented in Figure $1 \mathrm{~b}$ for a set of 6 vortices. This leads to a piecewise constant discretisation of the lifting line, but it is theoretically required in order to have a correct match between the local lift calculated from the Kutta formula and from the polar of the section. Non-linear curves for the 2D section coefficients of lift $\left(C l_{i}\right)$, drag $\left(C d_{i}\right)$, and moment about the quarter chord point $\left(\mathrm{Cm}_{i}\right)$, are also supposed to be given with respect to the angle of incidence. These curves will be denoted "polar curves" within this study.

The numerical iterative solution to calculate the circulation distribution $\Gamma=\left(\Gamma_{i}\right)_{i=1 \ldots n}$ is taken from Anderson (2011), but the calculation of the local effective angles of incidence has been adapted to the cases of wings which are non-straight and non-planar. The local circulation values are first initialized by an elliptical distribution along the wing span. At step $(p)$, starting from the $\left(\Gamma_{k}\right)_{k=1 \ldots n}^{(p)}$ values, for each point $M_{i}$ of the lifting line, the induced velocities by each horseshoe vortex $k$ are calculated with the Biot-Savart law and then summed, leading to the induced velocity $\vec{V}_{\text {indi }}^{(p)}$. Combined with the local free stream velocity $\vec{V}_{a i}=\vec{V}_{R W}-\left(\vec{V}_{K}+\vec{\Omega} \Lambda \overrightarrow{K M}_{i}\right)$, the effective wind is computed as $\vec{V}_{\text {eff } i}^{(p)}=\vec{V}_{a i}+\vec{V}_{\text {ind } i}^{(p)}$. This velocity is projected in the plane of the section $\left(\vec{x}_{s i}, \vec{y}_{s i}\right)$, leading to $\vec{V}_{p r j i}^{(p)}$ and to the section angle of effective incidence $\alpha_{p r j i}^{(p)}$ (see Fig. 1a). The updated values $\left(\Gamma_{k}\right)_{k=1 \ldots n}^{(p+1)}$ of the bound vortex strengths are finally calculated solving, for each section $i$, the equivalence between local lift calculated from the Kutta formula and calculated from the 2D polar of the section: $\left\|\rho \vec{V}_{\text {eff } i}^{(p)} \Lambda \Gamma_{i}^{(p+1)} \vec{t}_{i}\right\|=0.5 \rho V_{p r j i}^{(p) 2} c_{i} C l_{i}\left(\alpha_{p r j i}^{(p)}\right)$, which leads to $\Gamma_{i}^{(p+1)}=0.5 V_{p r j i}^{(p)} c_{i} C l_{i}\left(\alpha_{p r j i}^{(p)}\right)$. The values $\Gamma_{i}^{(p+1)}$ are ultimately updated by $\omega \Gamma_{i}^{(p+1)}+(1-\omega) \Gamma_{i}^{(p)}$ where $\omega \in[0 ; 1]$ is an under-relaxation factor. This whole process is repeated and, as expected, is found to converge according to a stopping criterion of the form $\left\|\Gamma^{(p+1)}-\Gamma^{(p)}\right\|<\left(10\left\|\Gamma^{(p+1)}\right\|+1\right) T o l$, Tol being a small user defined tolerance. Once convergence is reached, the lift, drag and torque of each section of the wing are post processed. Finally, these are summed vectorially, to obtain the global aerodynamic force and the global aerodynamic moment about the $K$ point.

Mesh convergence studies have been performed in order to estimate $\sigma$, the standard deviations of the global results, and then $\pm 2 \sigma$, their corresponding confidence intervals at $95 \%$. In the linear range, these are between $\pm 1 \%$ and $\pm 2 \%$ of the converged values. In the non-linear range, it is $\pm 4 \%$ for the lift coefficient and around $\pm 14 \%$ for the drag and the moment coefficients.

\section{Verification of the 3D lifting line method with RANSE simulations}

In order to validate the 3D non-linear lifting line method, its results have been compared to those obtained from 3D RANSE simulations performed with the generalist tool STAR$\mathrm{CCM}+{ }^{\circledR} .2 \mathrm{D}$ simulations have been used for convergence studies, but also to obtain the polar curves of the kite section, required to feed the lifting line model. $3 \mathrm{D}$ simulations have 


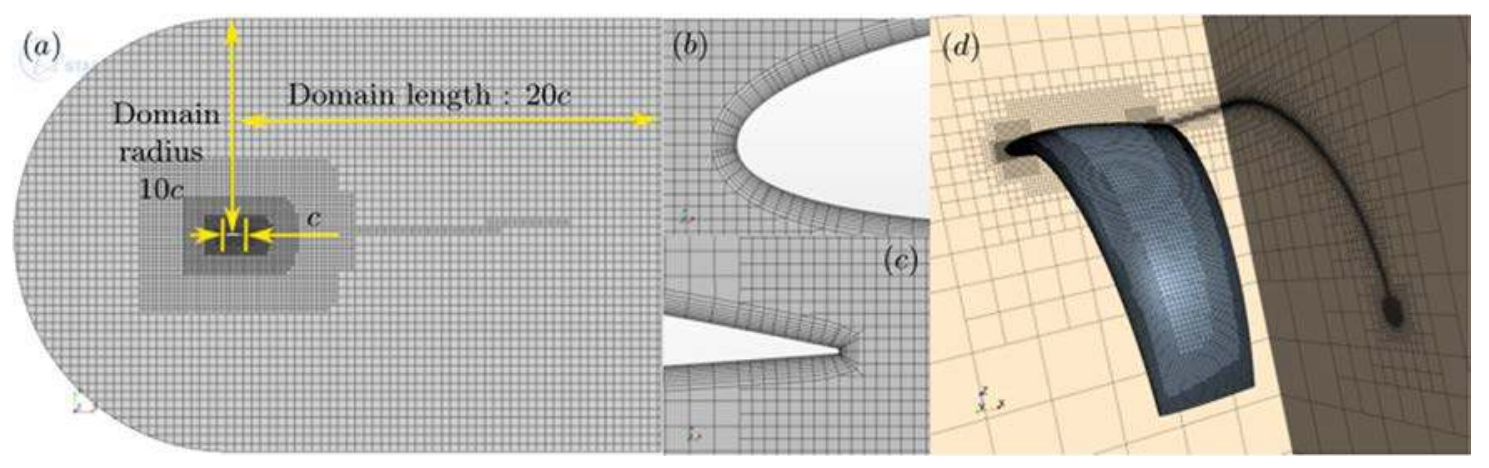

Fig. 2. Meshed computational domain: a: global view; b: leading edge; c: trailing edge; d: wake refinements.

then been used to calculate the aerodynamic efforts of a kite in translation, with respect to variations of the angle of incidence and the sideslip angle. Within this study, the chosen kite geometry is un-twisted and un-swept, with a semi-circular generatrix line of radius $1.5 \mathrm{~m}$ and a linear chord law varying from $1.0 \mathrm{~m}$ at the root section in the symmetry plane to $0.5 \mathrm{~m}$ at the tip. The kite section is kept constant along the span, and is defined by the NACA2412 ordinates (Abbott and von Doenhoff, 1959).

\subsection{General numerical set up}

Within this study, all the RANSE simulations are incompressible, steady and fully turbulent. The retained turbulence model is the two-equation $k-\varepsilon$ realizable model with a two-layer formulation for the wall treatment. The segregated flow solver is based on the SIMPLE algorithm, and a second-order discretisation scheme. The kite wing is fixed with respect to the computational domain, and the angle of incidence and the sideslip angle are modelled varying the inlet velocity direction. The Reynolds number is $3.1 \times 10^{6}$, based on the root section chord. The inlet turbulence intensity is set to $0.5 \%$ and the inlet turbulent viscosity ratio, $\mu_{T} / \mu$, where $\mu_{T}$ is the turbulent viscosity and $\mu$ is the molecular viscosity, is set to 1 .

The domain has been meshed using the trimmed cell mesher, which leads to predominantly hexahedral mesh (see Fig. 2). Mesh refinements of the wake have been prescribed in the mean free stream direction, from the trailing edge, and from the wing tips in order to partially capture the tip vortex (see Figs. $2 \mathrm{a}$ and $2 \mathrm{~d}$ ). The obtained mesh is coarse, of about $4.7 \times 10^{6}$ cells in $3 \mathrm{D}$, and leads to a mean value of $y^{+}$over the wing surface of about 35 in each simulated case. For all the simulated cases presented in the next section, converged steady solutions have effectively been obtained with the Navier-Stokes solver. The stopping criteria are based on the lift and drag coefficients, specifying a tolerance corresponding to the convergence towards zero of the non-dimensional residues over at least 5 decades. Mesh convergence studies have also been performed in order to estimate the following $\pm 2 \sigma$ confidence intervals at $95 \%$ for the global results: $\pm 3.7 \%$ for the lift, $\pm 7.7 \%$ for the drag, and $\pm 7.1 \%$ for the moment.

\subsection{Numerical results}

Firstly, 2D RANSE simulations have been carried out on the NACA2412 section, varying angle of incidence from $-8^{\circ}$ to $16^{\circ}$. The numerical results agree well with experimental ones from Abbott and von Doenhoff (1959) for each aerodynamic coefficient. The $2 \mathrm{D}$ polar curves used for the lifting line method have then been established, approaching these RANSE results at best in the least-squares sense, with piecewise polynomial curves. Secondly, systematic 3D RANSE simulations have been carried out for the validation of the lifting line method, firstly varying the angle of incidence for the kite wing at a fixed sideslip angle of $0^{\circ}$, and secondly varying the sideslip angle for the kite wing at a fixed incidence angle of $2^{\circ}$. In each case, the lift coefficient has been estimated from the aerodynamic force component orthogonal to the wind direction.

The results for the three aerodynamic coefficients are presented Figure 3, where the error bars are the estimated $\pm 2 \sigma$ confidence intervals at $95 \%$ for the RANSE and for the lifting line results.

For angle of incidence variations, the relative differences between the RANSE results and the lifting line method are 5\% for the lift coefficient and $12 \%$ for the moment coefficient, in average over the investigated range. For the drag coefficient, it is $5 \%$ at low angles of incidence, up to $20 \%$ for the higher angles of incidence.

For sideslip angle variations, for the three aerodynamic coefficients, the relative differences are roughly constant from $0^{\circ}$ to $15^{\circ}$, and nearly equal the corresponding values at $2^{\circ}$ of incidence and $0^{\circ}$ of sideslip. This is approximately $10 \%$ for the lift coefficient, and only of a few percent for the drag and moment coefficients. At $15^{\circ}$ of sideslip angle, the results start to differ more significantly for the drag coefficient. Following these results, the 3D non-linear lifting line results are satisfying over a large range of angle of incidence and of sideslip angle, typically included between $0^{\circ}$ and $15^{\circ}$.

\section{Conclusion}

In order to estimate the aerodynamic coefficients of a kite, a 3D non-linear lifting line method has been developed. This iterative method is adapted to wings that are neither straight 

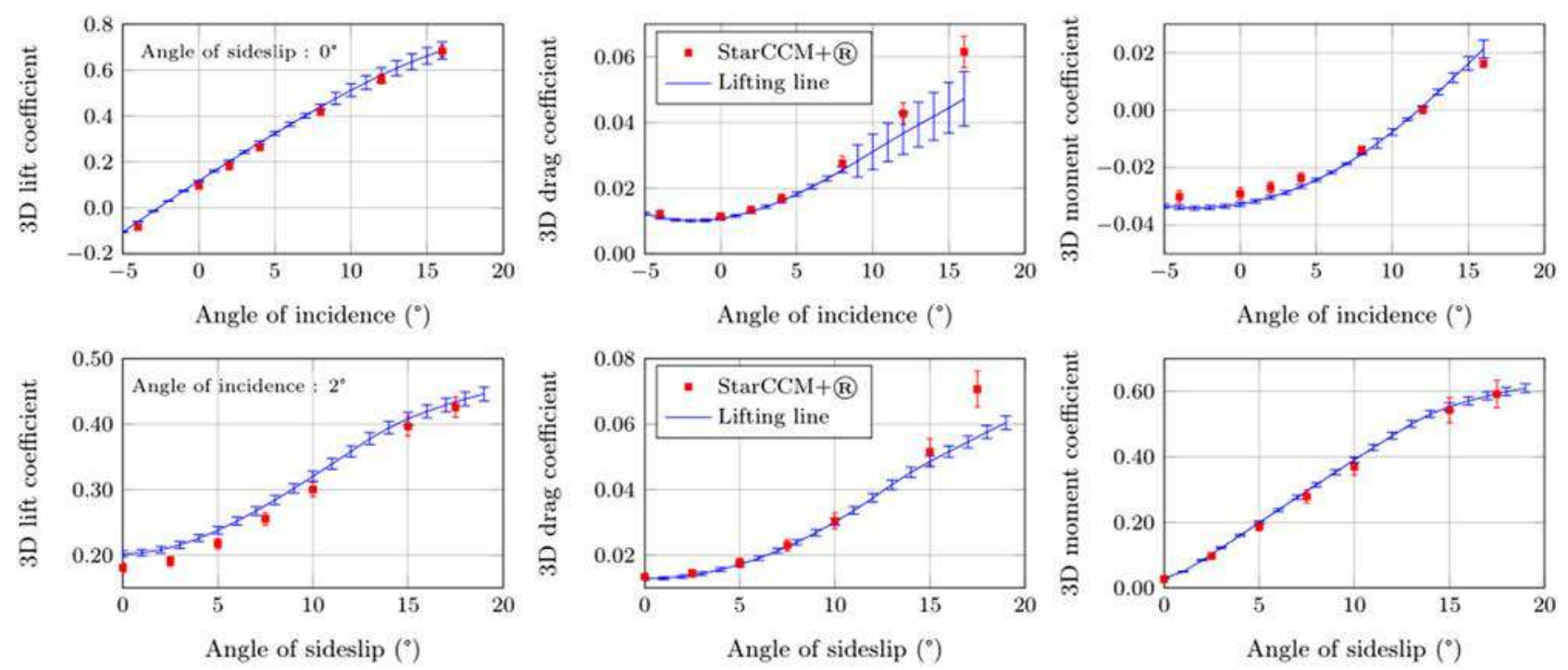

Fig. 3. Kite 3D aerodynamic coefficients with respect to the angle of incidence (top) or the angle of sideslip (bottom).

nor planar. It shows a low mesh dependency at low angles of incidence. The results of the lifting line method have been validated by a satisfactory comparison with $3 \mathrm{D}$ RANSE simulations, despite very different levels of approximation.

Acknowledgments. The authors are grateful to the French agency for energy development and control (ADEME) for the funding of this study.

\section{References}

Abbott IH, von Doenhoff AE. 1959. Theory of wing sections. New York: Dover Publications.

Anderson JD. 2011. Fundamentals of aerodynamics. Fifth ed. New York: McGraw-Hill.

Duport C, Leroux J-B, Roncin K, Jochum C, Parlier Y. 2016. Comparison of $3 \mathrm{D}$ non-linear lifting line method calculations with
3D RANSE simulations and application to the prediction of the global loading on a cornering kite. Actes des 15èmes Journées de l'Hydrodynamique. Available from http://website.ec-nantes.fr/ actesjh/images/15JH/Articles/duport.pdf.

Graf K, Hoeve AV, Watin S. 2014. Comparison of full 3D-RANS simulations with 2D-RANS/lifting line method calculations for the flow analysis of rigid wings for high performance multihulls. Ocean Eng 90: 49-61.

Katz J, Plotkin A. 2001. Low speed aerodynamics. Cambridge: Cambridge University Press.

Leloup R, Roncin K, Bles G, Leroux J-B, Jochum C, Parlier Y. 2013. Estimation of the lift-to-drag ratio using the lifting line method: Application to a leading edge inflatable kite. In: Ahrens U, Diehl M, Schmehl R, eds. Airborne Wind Energy. Green Energy and Technology. Berlin, Heidelberg: Springer.

Phillips WF, Snyder DO. 2000. Modern adaptation of Prandtl's classic lifting-line theory. $J$ Aircraft 37(4): 662-670. 